\title{
Gate-controlled spin-splitting in quantum dots with ferromagnetic leads in the Kondo regime
}

\author{
J. Martinek,,${ }^{1,3,6}$ M. Sindel,,${ }^{2}$ L. Borda, ${ }^{2,4}$ J. Barnaś,,${ }^{3,5}$ R. Bulla,${ }^{7}$ J. König, ${ }^{8}$ G. Schön, ${ }^{1}$ S. Maekawa, ${ }^{6}$ and J. von Delft ${ }^{2}$ \\ ${ }^{1}$ Institut für Theoretische Festkörperphysik, Universität Karlsruhe, 76128 Karlsruhe, Germany \\ 2 Physics Department and Center for NanoScience, LMU München, 80333 München, Germany \\ ${ }^{3}$ Institute of Molecular Physics, Polish Academy of Sciences, 60-179 Poznań, Poland \\ 4 Institute of Physics, TU Budapest, H-1521, Hungary \\ ${ }^{5}$ Department of Physics, Adam Mickiewicz University, 61-614 Poznań, Poland \\ ${ }^{6}$ Institute for Materials Research, Tohoku University, Sendai 980-857r, Japan \\ ${ }^{7}$ Theoretische Physik III, Elektronische Korrelationen und Magnetismus, Universität Augsburg, Augsburg, Germany \\ ${ }^{8}$ Institut für Theoretische Physik III, Ruhr-Universität Bochum, 44780 Bochum, Germany
}

(Dated: February 2, 2008)

\begin{abstract}
The effect of a gate voltage $\left(V_{g}\right)$ on the spin-splitting of an electronic level in a quantum dot (QD) attached to ferromagnetic leads is studied in the Kondo regime using a generalized numerical renormalization group technique. We find that the $V_{g}$-dependence of the QD level spin-splitting strongly depends on the shape of the density of states (DOS). For one class of DOS shapes there is nearly no $V_{g}$-dependence, for another, $V_{g}$ can be used to control the magnitude and sign of the spin-splitting, which can be interpreted as a local exchange magnetic field. We find that the spinsplitting acquires a new type of logarithmic divergence. We give an analytical explanation for our numerical results and explain how they arise due to spin-dependent charge fluctuations.
\end{abstract}

PACS numbers: 75.20.Hr, 72.15.Qm, 72.25.-b, 73.23.Hk

The manipulation of magnetization and spin is one of the fundamental processes in magneto-electronics and spintronics, providing the possibility of writing information in a magnetic memory [1], and also because of the possibility of classical or quantum computation using spin. In most situations this is realized by means of an externally applied, nonlocal magnetic field which is usually difficult to insert into an integrated circuit. Recently, it was proposed to control the magnetic properties, such as the Curie temperature of ferromagnetic semiconductors, by means of an electric field: In gated structures [2], due to the modification of carrier-density-mediated magnetic interactions, such properties can be modified by a gate voltage. In this Letter we propose to control the amplitude and sign of the spin-splitting of a quantum dot (QD) induced by the presence of ferromagnetic leads, only by using a gate voltage without further assistance of a magnetic field. To illustrate this effect we investigate the Kondo effect and its spin-splitting as a very sensitive probe of the spin state of the dot and the effective local magnetic field in the QD generated by exchange interaction with the ferromagnetic leads.

Recently, the possibility of the Kondo effect in a QD attached to ferromagnetic electrodes was widely discussed $[3,4,5,6,6,8,9]$, and it was shown, that the Kondo resonance is split and suppressed in the presence of ferromagnetic leads $7,[8]$. It was shown that this splitting can be compensated by an appropriately tuned external magnetic field, and the Kondo effect is thereby restored [7, 8]. In all previous studies of QDs attached to ferromagnetic leads $[\underline{3}, 4,[4,6,6,7,8,[9]$ an idealized, flat, spinindependent DOS with spin-dependent tunneling amplitudes was considered. However, since the spin-splitting arises from renormalization effects i.e. is a many-body effect, it depends on the full DOS-structure of the involved material, and not only on its value at the Fermi surface. In realistic ferromagnetic systems, the DOS shape is strongly asymmetric due to the Stoner splitting and the different hybridization between the electronic bands [1].

In this Letter we demonstrate that the gate voltage dependence of the spin-splitting of a QD level, resulting in a splitting and suppression of the Kondo resonance, is determined by the DOS structure and can lead to crucially different behaviours. We apply the numerical renormalization group (NRG) technique extended to handle bands of arbitrary shape. For one class of DOS-shapes, we find almost no $V_{g}$-dependence of the spin-splitting, while for another class the induced spin-splitting, which can be interpreted as the effect of a local exchange field, can be controlled by $V_{g}$. The spin-splitting can be fully compensated and its direction can even be reversed within this class. We explain the physical mechanism that leads to this behavior, which is related to the compensation of the renormalization of the spin-dependent QD levels induced by the electron-like and hole-like quantum charge fluctuations. Moreover we find that for the QD level close to the Fermi surface, the amplitude of the spin-splitting has a logarithmic divergence, indicating the many-body character of this phenomenon.

Model and method. - The Anderson model (AM) of a single level QD with energy $\epsilon_{0}$ and Coulomb interaction $U$, coupled to ferromagnetic leads, is given by

$$
H=\sum_{r k \sigma} \epsilon_{r k \sigma} c_{r k \sigma}^{\dagger} c_{r k \sigma}+\epsilon_{0} \sum_{\sigma} \hat{n}_{\sigma}+U \hat{n}_{\uparrow} \hat{n}_{\downarrow}
$$




$$
+\sum_{r k \sigma}\left(V_{r k} d_{\sigma}^{\dagger} c_{r k \sigma}+\text { h.c. }\right)-B S_{z} .
$$

Here $c_{r k \sigma}$ and $d_{\sigma}\left(\hat{n}_{\sigma}=d_{\sigma}^{\dagger} d_{\sigma}\right)$ are Fermi operators for electrons with momentum $k$ and spin $\sigma$ in the leads $(r=L / R)$, and in the QD, $V_{r k}$ is the tunneling amplitude, $S_{z}=\left(\hat{n}_{\uparrow}-\hat{n}_{\downarrow}\right) / 2$, and the last term denotes the Zeeman energy of the dot. The energy $\epsilon_{0}$ is experimentally controllable by $V_{g}\left(\epsilon_{0} \simeq V_{g}\right)$.

In order to discuss the gate voltage dependence of the QD level spin-splitting, we consider here a more realistic, both energy and spin dependent band structure $\left[\rho_{r \uparrow}(\omega) \neq \rho_{r \downarrow}(\omega)\right]$, violating p-h symmetry $\rho_{r \sigma}(\omega) \neq$ $\rho_{r \sigma}(-\omega)$, which leads to an energy dependent hybridization function $\Gamma_{r \sigma}(\omega)=\pi \sum_{k} \delta\left(\omega-\epsilon_{k \sigma}\right) V_{r k}^{2}=\pi \rho_{r \sigma}(\omega) V_{0}^{2}$, where we take $V_{r k}=V_{r}$ to be constant. We apply the NRG method [10, 11] extended to handle arbitrary DOS shapes and asymmetry. To this end, the standard logarithmic discretization of the conduction band is performed for each spin component separately, with the bandwidths, $D_{\uparrow}=D_{\downarrow}=D_{0}$, chosen such that the total spectral weight is included in $\left[-D_{0}, D_{0}\right]$ for all values of $V_{g}$ studied here (to avoid different systematic errors upon changing $V_{g}$ ).

Within each interval $\left[-\omega_{n},-\omega_{n+1}\right]$ and $\left[\omega_{n+1}, \omega_{n}\right]$ (with $\omega_{n}=D_{0} \Lambda^{-n}$ ) of the logarithmically discretized conduction band $(\mathrm{CB})$ the operators of the continuous $\mathrm{CB}$ are expressed in terms of a Fourier series. Even though we allow for a non-constant conduction electron DOS, it is still possible to transform the Hamiltonian such that the impurity couples only to the zeroth order component of the Fourier expansion of each interval [12]. Dropping the non-constant Fourier-components of each interval [10, 11] then results in a discretized version of the Anderson model with the continous spectrum in each interval replaced by a single fermionic degree of freedom (independently for both spin directions). Since we allow for an arbitrary DOS for each spin component $\sigma(\uparrow, \downarrow)$ of the CB this mapping needs to be performed for each $\sigma$ separately. This leads to the Hamiltonian:

$$
\begin{aligned}
& H=\sum_{\sigma} \epsilon_{\sigma} \hat{n}_{\sigma}+U \hat{n}_{\uparrow} \hat{n}_{\downarrow}+\sqrt{\xi_{0 \sigma} / \pi} \sum_{\sigma}\left[d_{\sigma}^{\dagger} f_{0 \sigma}+f_{0 \sigma}^{\dagger} d_{\sigma}\right] \\
& +\sum_{\sigma n=0}^{\infty}\left[\varepsilon_{n \sigma} f_{n \sigma}^{\dagger} f_{n \sigma}+t_{n \sigma}\left(f_{n \sigma}^{\dagger} f_{n+1 \sigma}+f_{n+1 \sigma}^{\dagger} f_{n \sigma}\right)\right],
\end{aligned}
$$

where $f_{n \sigma}$ are fermionic operators at the $n$th site of the Wilson chain, $\xi_{0 \sigma}=1 / 2 \int_{-D_{0}}^{+D_{0}} \Gamma_{\sigma}(\omega) d \omega, t_{n \sigma}$ denotes the hopping matrix elements, and $\epsilon_{\sigma}=\epsilon_{0}-B S_{z}$. The absence of particle-hole symmetry leads to the appearance of non-zero on-site energies, $\varepsilon_{n \sigma}$ along the chain. In this general case no closed expression for the matrix elements $t_{n \sigma}$ and $\varepsilon_{n \sigma}$, both depending on the particular structure of the DOS via $\Gamma_{\sigma}(\omega)$, is known, therefore they have to be determined recursively. This requires rather advanced numerical methods, due to the exponentially fast decay of $t_{n \sigma}$ and $\varepsilon_{n \sigma}$ along the chain [13].

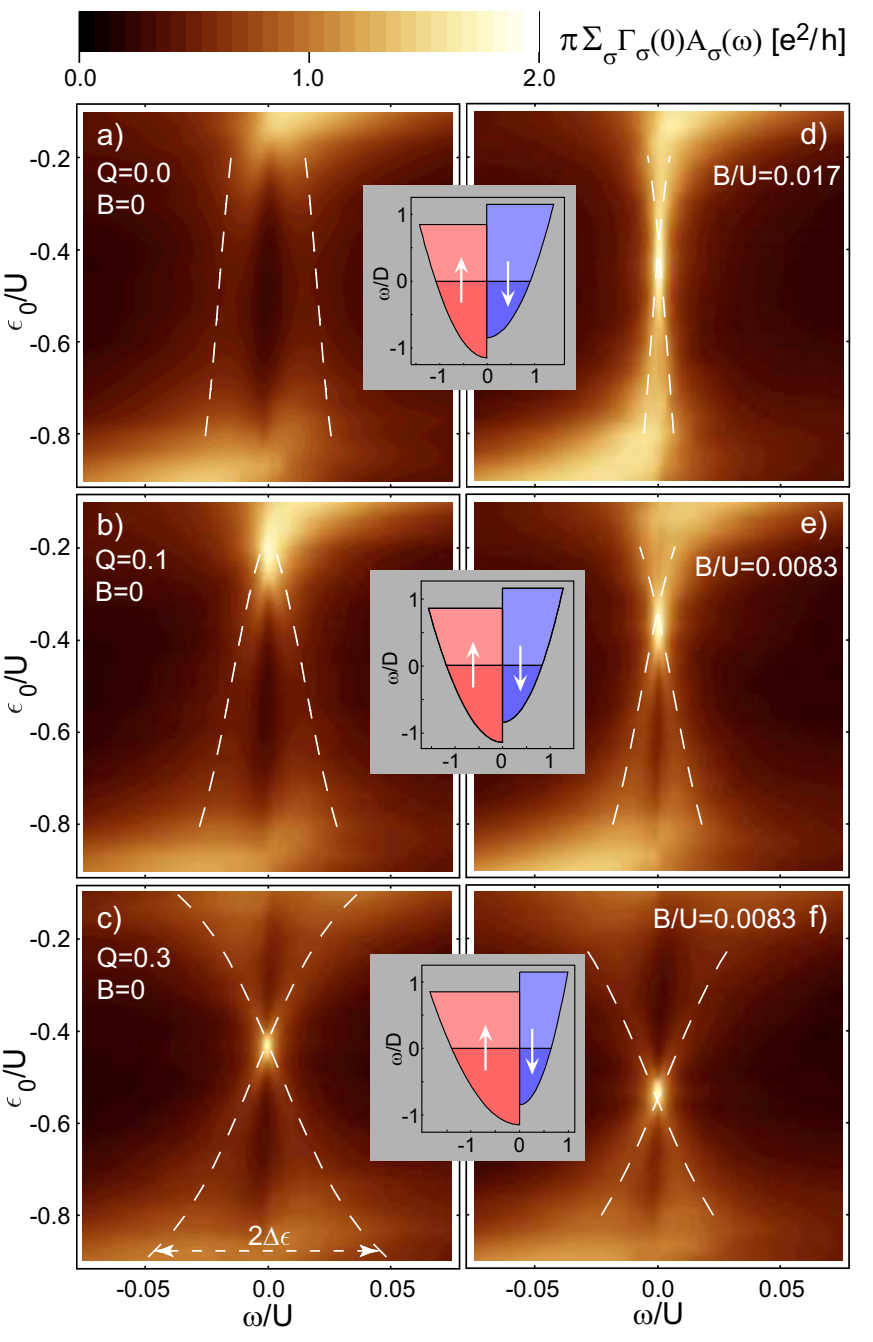

FIG. 1: (color online). $V_{g}$-dependence of the spin-splitting: Normalized spectral function $\pi \sum_{\sigma} \Gamma_{\sigma}(0) A_{\sigma}(\omega)$ as a function of energy $\omega$ and gate voltage $\epsilon_{0}$, for the three different DOS shapes (depicted in insets) characterized by a different $Q$, which modifies both the spin and p-h asymmetry: (a-c) for magnetic filed $B=0$, (d) $B / U=0.017$, (e), and (f) $B / U=$ 0.0083. The white dashed lines are obtained using Eq. (3). Here $U=0.12 D_{0}, \pi V_{0}^{2}=U D / 6, \Delta=0.15 D$ and $T=0$. Inset: the scheme of the parabolic DOS shape for spin $\uparrow$ (red) and $\downarrow$ (blue).

This method allows one to calculate the level occupation $n_{\sigma} \equiv\left\langle\hat{n}_{\sigma}\right\rangle$ and the spin-resolved single-particle spectral density $A_{\sigma}(\omega)=-\frac{1}{\pi} \operatorname{Im} \mathcal{G}_{\sigma}^{\mathrm{r}}(\omega)$, where $\mathcal{G}_{\sigma}^{\mathrm{r}}(\omega)$ denotes a retarded Green's function. For symmetric coupling $\left[\Gamma_{L \sigma}(\omega)=\Gamma_{R \sigma}(\omega)\right]$ the spin-resolved conductance takes the form $G_{\sigma}=\pi \frac{e^{2}}{h} \int_{-\infty}^{+\infty} d \omega \Gamma_{\sigma}(\omega) A_{\sigma}(\omega)\left(-\frac{\partial f(\omega)}{\partial \omega}\right)$ where $f(\omega)$ is the Fermi function.

Spectral function and conductance. - Here, we focus our attention on $T=0$ properties. We have analyzed several types of DOS shapes and found three typical classes of the $V_{g}$-dependence of the Kondo resonance splitting. Since our method enables us to per- 
form NRG calculations for arbitrary band-shapes, we decide to choose an example which turns out to encompass all three classes, namely $\rho_{\sigma}(\omega)=\frac{1}{2} \frac{3 \sqrt{2}}{8} D^{-3 / 2}(1+$ $\sigma Q) \sqrt{\omega+D+\sigma \Delta}$, where $\omega \in[-D-\sigma \Delta, D-\sigma \Delta]$, $D_{0}=D+\Delta,[\sigma \equiv 1(-1)$ for $\uparrow(\downarrow)]$, a square-root shape DOS equivalent to a parabolic band (as for free electrons) with Stoner splitting $\Delta[14]$, and some additional spin asymmetry $Q$, which modifies the amplitude of the DOS [see Fig. 1(insets)].

In Fig. 1 we present the weighted spectral function $\tilde{A}(\omega) \equiv \pi e^{2} / h \sum_{\sigma} \Gamma_{\sigma}(0) A_{\sigma}(\omega)$, normalized such that for $\omega=0$ it corresponds to the linear conductance $G=\tilde{A}(0)$, as a function of energy $\omega$ and $\epsilon_{0}$. We focus on a narrow energy window around the Fermi surface where the Kondo resonance appears; charge resonances are visible when $\epsilon_{0}$ or $U+\epsilon_{0}$ approach the Fermi surface, namely at energies $\epsilon_{0} / U \gtrsim-0.1$ or $\lesssim-0.9$. Although the NRG method is designed to calculate equilibrium transport, one can still roughly deduce, from the spin-splitting of the Kondo resonance of the equilibrium spectral function $\tilde{A}(\omega)$, the splitting of the zero-bias anomaly $\Delta V$ in the non-equilibrium conductance $G(V)$, since $e \Delta V \sim 2 \Delta \epsilon[]$ $\left(\Delta \epsilon \equiv \tilde{\epsilon}_{\uparrow}-\tilde{\epsilon}_{\downarrow}\right.$ is the splitting of the renormalized levels).

We present $\tilde{A}(\omega)$ for three DOS shapes depicted in the insets of Fig. 11 (i) $Q=0$ (a,d), (ii) $Q=0.1$ (b,e), and (iii) $Q=0.3$ (c,f), with $2 \Delta=0.3 D$ [15], leading to the three typical behaviors. Here the parameter $Q$ tunes the spin and p-h asymmetry [see the definition of $\rho_{\sigma}(\omega)$ ] resulting in different behaviours (for a detailed discussion see the last section). For (i) we find nearly any $\epsilon_{0}$-dependence of the spin-splitting; for (ii), a strong $\epsilon_{0^{-}}$ dependence without compensation of the spin-splitting (i.e. no crossing), and for (iii) a strong $\epsilon_{0}$-dependence with a compensation (i.e. a crossing) and a change of the direction of the QD magnetization. The compensation (crossing) corresponds to the very peculiar situation where the Kondo effect (strong coupling fixed point) can be recovered in the presence of ferromagnetic leads without any external magnetic field. A behavior as presented in Fig. 1(a,b) was recently observed experimentally [16, 17], where indeed a variation of the gate voltage results in two split conduction lines $G\left(V, V_{g}\right)$ which are parallel for one case and converging for the other case, similar to our findings.

Effect of a magnetic field. - In Fig. 1(d,e,f) we show how a magnetic field $B$ modifies the results of Fig. 11 (a,b,c): in (i) the spin splitting can be compensated at a particular magnetic field $B_{\text {comp }}$ (here $B_{\text {comp }} / U=$ 0.017 ) and the Kondo effect is visible in a wide range of $\epsilon_{0}$; for (ii), at $B / U=0.0083$, the Kondo effect is recovered only at one particular $\epsilon_{0}$-value, which depends on the applied magnetic field; case (iii) shows that the crossing point shifts with $B$. Since $B_{\text {comp }}$ can be viewed as a measure of the zero-field splitting, $\Delta \epsilon\left(B=0, \epsilon_{0}\right) \simeq$ $-B_{\text {comp }}\left(\epsilon_{0}\right)$, the $\epsilon_{0}$-dependence of $\Delta \epsilon$ can be measured

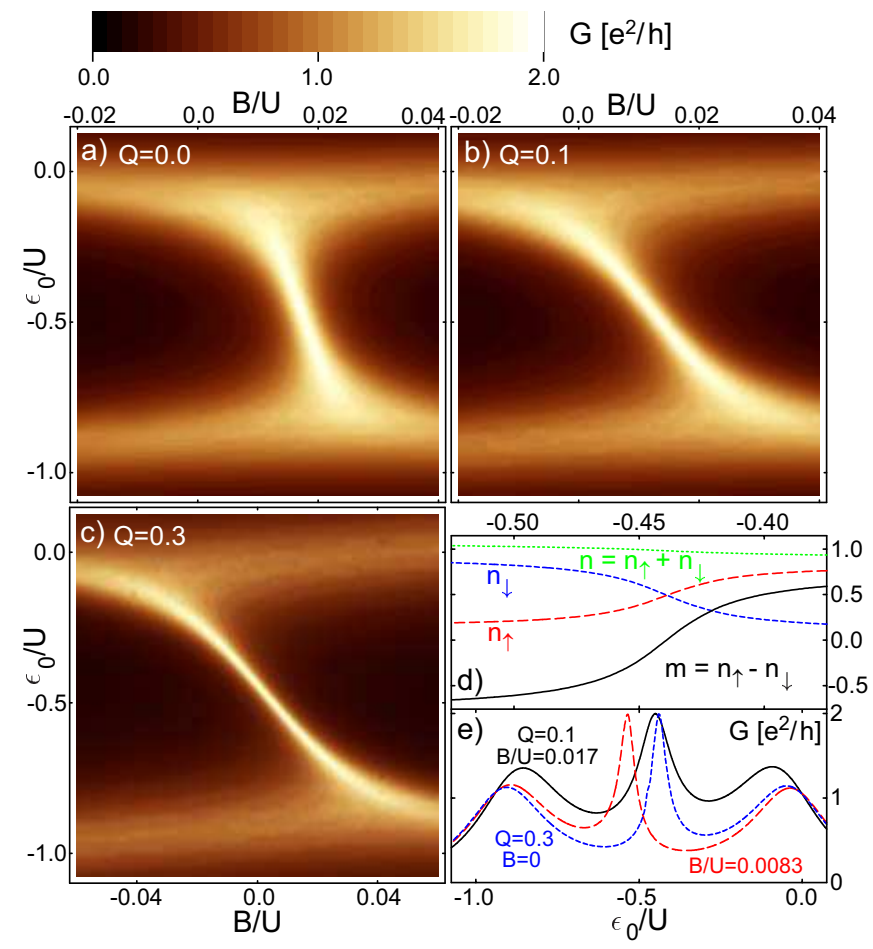

FIG. 2: (color online). The QD's linear conductance $G$ as a function of gate voltage $\epsilon_{0}$ and external magnetic field $B$ for the DOS shapes (a), (b), and (c) as for Fig. 1(a), (b), and (c) respectively. (d) Spin-dependent occupancy $n_{\sigma}$ of the dot level as a function of gate voltage $\epsilon_{0}$ for the DOS shape as in Fig. 1(c) and $B=0$. (d) The $\epsilon_{0}$-dependence of the total occupancy of the $\operatorname{dot} n$ and magnetization $m$ for the situation from Fig. 1(c). (e) The conductance $\mathrm{G}$ for the situations from Fig. 1 (c - dashed), (d - solid), and (f - long dashed). Parameters $U, \Gamma$, and $T$ as in Fig. 1

by studying that of $B_{\text {comp }}$, for which one needs to measure the linear conductance $G\left(\epsilon_{0}, B\right)$ as a function of both $B$ and $\epsilon_{0}$. In Fig. 2(a-c) we plot $G\left(\epsilon_{0}, B\right)$ for the three bands of Fig. 11. The two horizontal ridges (resonances) in Fig. 2(a-c) correspond to quantum charge fluctuations (broadened QD level) of width $\sim \Gamma$. The lines with finite slope in Fig. 2(a-c) reflect the restored Kondo resonance and hence map out the $\epsilon_{0}$-dependence of $B_{\text {comp }}\left(\epsilon_{0}\right)=-\Delta \epsilon\left(\epsilon_{0}\right)$ when the magnetic field compensates the spin-splitting. Interestingly the spin-splitting and the corresponding $B_{\text {comp }}$ tend to diverge $(|\Delta \epsilon| \rightarrow \infty)$ when approaching the charging resonance, as is best visible in Fig. 2(c).

Such a finite slope in $G\left(\epsilon_{0}, B\right)$ was observed for a singlet-triplet transition Kondo effect in a two level QD (Fig.2(d) Ref. [18]). The corresponding transition leads to a characteristic maximum in the valley between two charging resonances (Fig.3(c) Ref. [18]), similarly as in our Fig. 2(e). In that system the effective spin asymmetry (assumed by our model) is realized by the asymmetry in the coupling of two QD levels [19].

In Fig. 2(d) we show how the occupation $n_{\sigma}$ and the 
magnetic moment (spin) of the QD $m=n_{\uparrow}-n_{\downarrow}=2\left\langle S_{z}\right\rangle$ change as a function of $\epsilon_{0}$ for the situation of Fig. 11(c). One finds that even though $B=0$, it is possible to control the level spin-splitting of the QD, i.e. its spin, and thereby change the average spin direction of the QD from the parallel to anti-parallel alignment w.r.t. the lead's magnetization. This opens the possibility of controlling the QD's spin state by means of a gate voltage without further need of an external magnetic field, which is difficult to apply locally in practical devices.

Perturbative analysis. - One can understand the behavior presented in Fig. 1(a-c) by using Haldane's scaling method [20], where charge fluctuations are integrated out. This leads to a spin-dependent renormalization of the QD's level position $\tilde{\epsilon}_{\sigma}$ and a level broadening $\Gamma_{\sigma}$. In contrast to Ref. 7] we consider here the case of finite Coulomb interactions $U<\infty$, which means that also the doubly occupied state $|2\rangle$ is of importance. The spinsplitting is then given by $\Delta \epsilon \equiv \delta \epsilon_{\uparrow}-\delta \epsilon_{\downarrow}+B$, where

$\delta \epsilon_{\sigma} \simeq-\frac{1}{\pi} \int d \omega\left\{\frac{\Gamma_{\sigma}(\omega)[1-f(\omega)]}{\omega-\epsilon_{\sigma}}+\frac{\Gamma_{-\sigma}(\omega) f(\omega)}{\epsilon_{-\sigma}+U-\omega}\right\}$

The first term in the curly brackets corresponds to electron-like processes, namely charge fluctuations between a single occupied state $|\sigma\rangle$ and the empty $|0\rangle$ one, and the second term to hole-like processes, namely charge fluctuations between the states $|\sigma\rangle$ and $|2\rangle$. The amplitude of the charge fluctuations is proportional to $\Gamma$, which for $\Gamma \gg T$ determines the width of QD's levels. Eq. (3) shows that $\Delta \epsilon$ depends on the shape of $\Gamma_{\sigma}(\omega)$ for all $\omega$, not only on its value at the Fermi surface. The dashed lines in Fig. 1(a-c) show $\pm \Delta \epsilon$ as a function of $\epsilon_{0}$ [from Eq. (3)] for the same set of parameters as in the NRG calculation, and are in good agreement with the position of the (split) Kondo resonances observed in the latter. Eq. (3) shows that the dramatic changes observed in Fig. 11 upon changing $Q$ are due to the modification of the $\mathrm{p}-\mathrm{h}$ and spin asymmetry.

Eq. (3) predicts that even for systems with spinasymmetric bands $\Gamma_{\uparrow}(\omega) \neq \Gamma_{\downarrow}(\omega)$, the integral can give $\Delta \epsilon=0$, which corresponds to a situation where the renormalization of $\epsilon_{\sigma}$ due to electron-like processes are compensated by hole-like processes. An example is a system consisting of p-h symmetric bands, $\Gamma_{\sigma}(\omega)=\Gamma_{\sigma}(-\omega)$, for which there is no splitting of the Kondo resonance $(\Delta \epsilon=0)$ for the symmetric point, $\epsilon_{0}=-U / 2$. For real systems p-h symmetric bands cannot be assumed, however the compensation $\Delta \epsilon=0$ is still possible, as in Fig. 1(c). Eq. (3) also shows that the characteristic energy scale of the spin-splitting is given by $\Gamma$ rather than by the Stoner splitting $\Delta(\Delta \gg \Gamma)$, since the states far from the Fermi surface enter Eq. (3) only with a logarithmic weight. However, the Stoner splitting introduces a strong p-h asymmetry, so it can influence the character of gate voltage dependence significantly.
For a flat band $\Gamma_{\sigma}(\omega)=\Gamma_{\sigma}$, Eq. (3) can be integrated analytically. For $D_{0} \gg U,\left|\varepsilon_{0}\right|$ one finds: $\Delta \epsilon \simeq$ $(P \Gamma / \pi) \operatorname{Re}\left[\phi\left(\epsilon_{0}\right)-\phi\left(U+\epsilon_{0}\right)\right]$, where $P \equiv\left(\Gamma_{\uparrow}-\Gamma_{\downarrow}\right) / \Gamma$, $\phi(x) \equiv \Psi\left(\frac{1}{2}+i \frac{x}{2 \pi T}\right)$, and $\Psi(x)$ denotes the digamma function. For $T=0$, the spin-splitting is given by

$\Delta \epsilon \simeq(P \Gamma / \pi) \ln \left(\left|\epsilon_{0}\right| /\left|U+\epsilon_{0}\right|\right)$,

showing a logarithmic divergence for $\epsilon_{0} \rightarrow 0$ or $U+\epsilon_{0} \rightarrow$ 0 . Since any sufficiently smooth DOS can be linearized around the Fermi surface, this logarithmic divergence occurs quite universally, as can be observed in log-linear versions (not shown) of Fig. 2(a-c). For finite temperature $(T>0)$ the logarithmic divergence for $\epsilon_{0} \rightarrow 0$ or $\epsilon_{0} \rightarrow-U$ is cut off, $\Delta \epsilon \simeq-\frac{1}{\pi} P \Gamma\left[\Psi\left(\frac{1}{2}\right)+\ln \frac{2 \pi T}{U}\right]$, which is also important for temperatures $T \ll T_{\mathrm{K}}$.

In conclusion, we demonstrated, using the extended NRG technique for general band shapes, the possibility of controlling the local exchange field and thereby the spin-splitting in a QD attached to ferromagnetic leads by means of the gate voltage. A new type of the logarithmic divergence of the QD's level spin-splitting was found, and attributed to spin-dependent charge fluctuations.

We thank T. Costi, L. Glazman, W. Hofstetter, B. Jones, C. Marcus, J. Nygård, A. Pasupathy, D. Ralph, A. Rosch, M. Vojta, and Y. Utsumi for discussions. This work was supported by the DFG under the CFN, 'Spintronics' RT Network of the EC RTN2-2001-00440, and Project OTKA T034243.

[1] S. Maekawa and T. Shinjo, Spin Dependent Transport in Magnetic Nanostructures, Taylor \& Francis (2002).

[2] H. Ohno et al., Nature (London) 408, 944 (2000). D. Chiba et al., Science 301, 943 (2003).

[3] N. Sergueev et al., Phys. Rev. B 65, 165303 (2002).

[4] P. Zhang et al., Phys. Rev. Lett. 89, 286803 (2002).

[5] B. R. Bułka et al., Phys. Rev. B 67, 024404 (2003).

[6] R. Lopez et al., Phys. Rev. Lett. 90, 116602 (2003).

[7] J. Martinek et al., Phys. Rev. Lett. 91, 127203 (2003).

[8] J. Martinek et al., Phys. Rev. Lett. 91, 247202 (2003).

[9] In Ref. [M. S. Choi et al., Phys. Rev. Lett. 92, 056601 (2004)] among other results the $V_{g}$-dependence of a QD coupled to ferromagnetic leads with a flat ( $\mathrm{p}-\mathrm{h}$ symmetric) DOS has been presented. However, the role of charge fluctuations in the absence of a spin-splitting, a sign change and a logarithmic divergence in the spin-splitting went unnoticed.

[10] K.G. Wilson, Rev. Mod. Phys. 47773 (1975); T. A. Costi et al., J. Phys.: Cond. Mat. 6, 2519 (1994).

[11] A. C. Hewson, The Kondo Problem to Heavy Fermions, Cambridge Univ. Press (1993).

[12] R. Bulla et al., J. Phys.: Condens. Matter 9, 10463 (1997).

[13] For more details see [N.-T. Tong et al., in preparation].

[14] K. Yosida, Theory of Magnetism, Springer (1996).

[15] This value is in the range for typical ferromagnetic metals like $N i, C o$, and $F e$ [D. A. Papaconstantopoulos, ed., Handbook of the Band Structure of Elemental Solids, Plenum Press (1986)].

[16] J. Nygård and C. M. Markus (private commun.). 
[17] D. Ralph and A. Pasupathy (private commun.).

[18] S. Sasaki et al., Nature (London) 405, 764 (2000).

[19] D. Boese et al., Phys. Rev. B 64, 125309 (2001).
[20] F. D. M. Haldane, Phys. Rev. Lett. 40, 416 (1978). 